\title{
BMJ Open Temporal trends in hyperuricaemia among adults in Wuhan city, China, from 2010 to 2019: a cross-sectional study
}

\author{
Zhengce Wan, ${ }^{1}$ Lulu Song, ${ }^{2}$ Liu Hu, ${ }^{1}$ Xiaomei Lei, ${ }^{1}$ Yuancheng Huang, ${ }^{1}$ \\ Yongman LV (i) ${ }^{1}$
}

To cite: Wan Z, Song L, Hu L, et al. Temporal trends in hyperuricaemia among adults in Wuhan city, China, from 2010 to 2019: a crosssectional study. BMJ Open 2021;11:e043917. doi:10.1136/ bmjopen-2020-043917

- Prepublication history and additional materials for this paper is available online. To view these files, please visit the journal online (http://dx.doi. org/10.1136/bmjopen-2020043917).

LH and YL contributed equally.

Received 17 August 2020 Revised 10 February 2021 Accepted 15 March 2021

Check for updates

(c) Author(s) (or their employer(s)) 2021. Re-use permitted under CC BY-NC. No commercial re-use. See rights and permissions. Published by BMJ.

${ }^{1}$ Health Management Center, Tongji Hospital, Tongji Medical College, Huazhong University of Science and Technology, Wuhan, China

${ }^{2}$ Department of Maternal and Child Health, School of Public Health, Tongji Medical College, Huazhong University of Science and Technology, Wuhan, China

Correspondence to

Dr Yongman Lv; Ivyongman@126.com and

Mrs Liu Hu;

huliu01230@163.com

\section{ABSTRACT}

Objectives Hyperuricaemia is a risk factor for gout attacks, kidney damage and cardiovascular events. Evidence on the trends in hyperuricaemia burden in Wuhan city, China, was limited. The present study aimed to estimate the prevalence of and a decade trend in hyperuricaemia in Wuhan city.

Design Cross-sectional study

Setting Health Management Center of Tongji Hospital. Participants A total of 732527 adult participants from the general population who took a physical examination in the Health Management Center between 2010 and 2019.

Main outcome measures Prevalence of and trends in hyperuricaemia.

Results The overall prevalence of hyperuricaemia was $25.8 \%$ (36.6\% in men and $10.8 \%$ in women) in 2019 . The hyperuricaemia prevalence and serum uric acid (SUA) levels were significantly higher in young men, old women and participants with obesity, hypertension, diabetes or dyslipidaemia $(p<0.05)$. SUA levels among men and women gradually increased from 358.0 (313.0407.0) umol/L and 250.0 (217.0-288.0) umol/L in 2010 to $388.0(338.0-445.2) \mathrm{umol} / \mathrm{L}$ and $270.0(233.0-314.0)$ umol/L in 2019, respectively, $p<0.05$. From 2010 through 2019, hyperuricaemia prevalence significantly increased in each age category and it increased most sharply among participants aged $20-39$ years. The multivariate-adjusted prevalence among men was $26.1 \%$ (25.4\% to $26.7 \%$ ) in $2010,30.9 \%$ (30.4\% to $31.4 \%$ ) in 2015 and $34.4 \%$ $(34.1 \%$ to $34.8 \%)$ in 2019 , while among women it was $5.8 \%(5.4 \%$ to $6.2 \%)$ in $2010,7.2 \%(6.9 \%$ to $7.5 \%)$ in 2015 and $10.1 \%$ (9.9\% to $10.3 \%)$ in 2019 .

Conclusions Hyperuricaemia was highly prevalent among adults in Wuhan city. More attention should be paid to the increasing burden of hyperuricaemia, especially for those at higher risks.

\section{INTRODUCTION}

Serum uric acid (SUA) is the final product of purine nucleotides metabolism. The overproduction of SUA, as well as its inefficient renal or gut excretion, can consequently lead to hyperuricaemia. Hyperuricaemia is generally recognised as the major contributor to the onset of gout. ${ }^{1}$ Moreover, many studies have demonstrated a positive association of hyperuricaemia or a high level of SUA with

\section{Strengths and limitations of this study}

This study included a large sample size of participants (more than 730000 adults) from the general population, which made our findings more convincible.

- This study first estimated the prevalence of and trends in hyperuricaemia over the recent decade (2010-2019) among adults in Wuhan city.

- The multivariate logistic model was used to correct selection biases and confounding biases as possible by adjusting for potential confounders.

- Since participants in the present study were recruited from a health management centre, selection biases could not be avoided.

increased all-cause mortality and several chronic diseases such as chronic kidney disease, cardiovascular events, reduced pulmonary function, obesity, glucose metabolism, dyslipidaemia, hypertension and metabolic syndrome. $^{2-9}$ These diseases accounted for a large part of global deaths and disabilityadjusted life-year losses, ${ }^{10}{ }^{11}$ resulting in a particular urgency to prevent and control hyperuricaemia.

Previous studies have documented an increasing trend of hyperuricaemia prevalence in the past few decades across the world, especially in western countries. ${ }^{12-14}$ Additionally, hyperuricaemia is reported to be more prevalent in developed countries than in developing countries. ${ }^{15}$ The prevalence among US adults was substantial (20.2\% in men and $20.0 \%$ in women) during 2015-2016. ${ }^{16}$ In China, the prevalence estimated from national surveys were $8.4 \%$ in $2009-2010,{ }^{17} 13.0 \%$ in $2007-2011^{18}$ and $6.4 \%$ among the middle-aged and elderly in 20112012. ${ }^{19}$ Besides, a meta-analysis of 38 studies from mainland China reported that the pooled prevalence of hyperuricaemia among Chinese adults was $13.3 \%$ in $2000-2014 .^{20}$ Obviously, the data above were not able to 
clearly illustrate the trend of hyperuricaemia prevalence among Chinese adults.

China is a large developing country with unbalanced economic development and marked regional differences. Hyperuricaemia prevalence among Chinese adults varied greatly by geographical regions, with a report of $18.6 \%$ in south China, $12.9 \%$ in east China, $13.9 \%$ in southwest China, $10.3 \%$ in northwest China, $10.1 \%$ in northeast China and $13.2 \%$ in north China. ${ }^{20}$ Noteworthily, evidence on hyperuricaemia burden in central China was limited. Wuhan, the capital city of Hubei province in central China, was characterised by rapid economic growth and urbanisation in the recent decade. Its gross domestic products per capita increased from ¥50 117 in 2009 to ¥123831 in $2017 .^{21}{ }^{22}$ Studies from developing countries showed that a rapidly growing economy in the short-term and subsequently changed lifestyles would increase the risk of several metabolic disorders. ${ }^{23} 24$

To date, few studies have investigated a decade trend in hyperuricaemia burden in Wuhan city. Therefore, we performed a large cross-sectional study to estimate the sex-specific prevalence of and trends in hyperuricaemia among the general adults from 2010 through 2019. This general population-based study used data collected from consecutive adults who underwent a health check-up in a health management centre.

\section{METHODS}

\section{Study population}

We conducted a cross-sectional study, of which consecutive participants who had a health check-up in the Health Management Center of Tongji Hospital in Wuhan city, China, between 1 January 2010 and 31 December 2019 were included. All the participants came from the general population. Most of them were urban citizens working in government organisations or large enterprises. Each participant completed the basic items of the physical examination including demographic information collection, biochemical tests and anthropometric measurements. Online supplemental figure 1 showed the procedures for selecting participants in the study. A total of 732527 participants aged $\geq 20$ years were recruited. This age range was selected in accordance with several previous studies. ${ }^{125}$ After excluding participants with missing data, we included 676478 participants. As reduced renal function may affect uric acid excretion, we further excluded 5027 participants who had an estimated glomerular filtration rate (eGFR) of less than $60 \mathrm{~mL} / \mathrm{min} / 1.73 \mathrm{~m}^{2}$. Finally, 671451 participants were included in the analyses. Due to the lack of information on ID card number, there existed some participants who underwent health check-ups for years during the study period.

We used the Strengthening the Reporting of Observational Studies in Epidemiology cross-sectional reporting guidelines in the present study. ${ }^{26}$

\section{Assessment of main variables}

Overnight fasting blood samples were collected to measure the biochemical variables. SUA concentrations were measured by the enzymatic colorimetry using an automated analyser (Roche cobas 8000, Basel, Switzerland) according to standard laboratory process of quality control. Additionally, the method of SUA measurement did not change during the whole study period and the laboratory quality assessment was reviewed. The intra-assay and inter-assay coefficients of variation were $4.25 \%$ and $<5.7 \%$ at level $1(210 \mathrm{umol} / \mathrm{L}),<4.25 \%$ and $<5.7 \%$ at level $2(572 \mathrm{umol} / \mathrm{L})$. In the current study, hyperuricaemia was defined according to SUA levels: men $\geq 416 \mathrm{umol} / \mathrm{L}$ $(7 \mathrm{mg} / \mathrm{dL})$ and women $\geq 357 \mathrm{umol} / \mathrm{L}(6 \mathrm{mg} / \mathrm{dL}) .{ }^{27} 28$ Participants were asked to wear light clothes and bare foot before their height and weight were measured. The body mass index (BMI) was calculated as weight in kilograms divided by height in metres squared. For Chinese adults, obesity was defined as BMI $\geq 28 \mathrm{~kg} / \mathrm{m}^{2}$ and overweight was defined as BMI $\geq 24 \mathrm{~kg} / \mathrm{m}^{2} .{ }^{29}$ Blood pressure was measured by trained nurses using electronic sphygmomanometers (HBP-9020; OMRON, Dalian, China) in a seated position after participants had rested for at least $5 \mathrm{~min}$. Hypertension was identified if any of the following criteria was satisfied: systolic blood pressure $\geq 140 \mathrm{~mm} \mathrm{Hg}$, or diastolic blood pressure $\geq 90 \mathrm{~mm} \mathrm{Hg}$, or use of antihypertensive medication, or self-reported physician diagnosis of hypertension. Diabetes was defined as fasting blood glucose $\geq 7.0 \mathrm{mmol} / \mathrm{L}$, or use of antidiabetic medication, or selfreported physician diagnosis of diabetes. Dyslipidaemia was diagnosed according to the 2016 Chinese Guideline for the Management of Dyslipidemia in Adults ${ }^{30}$ if any of the following criteria was satisfied: total cholesterol $\geq 6.22 \mathrm{mmol} / \mathrm{L}$, triglyceride $\geq 2.26 \mathrm{mmol} / \mathrm{L}$, low density lipoprotein cholesterol $\geq 4.14 \mathrm{mmol} / \mathrm{L}$, high density lipoprotein cholesterol $<1.04 \mathrm{mmol} / \mathrm{L}$. Information on age and sex was provided by the participants.

\section{Patient and public involvement}

Patients or the public were not involved in the design, or conduct, or reporting, or dissemination plans of our research. Patients or the public were not invited to contribute to the writing or editing of this article for readability or accuracy.

\section{Statistical analysis}

Due to the marked difference in SUA levels between men and women, the sex-specific prevalence of and trends in hyperuricaemia were estimated. Categorical variables were expressed in percentages, whereas continuous variables were reported as means \pm SD for normally distributed data or median (IQR) for skewed data. The normality of data is evaluated by Kolmogorov-Smirnov test. We used data collected in 2019 to estimate SUA levels, the hyperuricaemia prevalence and their $95 \%$ CIs, stratified by sex (male or female), age (20-29, 30-39, 40-49, 50-59, 60-69 or $\geq 70$ years $)$, BMI $\left(<24,24-27.9\right.$ or $\geq 28 \mathrm{~kg} / \mathrm{m}^{2}$, the cut-off values of overweight and obesity for Asian), hypertension 
(yes or no), diabetes (yes or no) and dyslipidaemia (yes or no). In this part, the prevalence of hyperuricaemia was compared using the Cochran-Armitage test for trend. SUA levels were compared using Kruskal-Wallis test.

Sex-specific and age-specific trends in hyperuricaemia from 2010 through 2019 were then analysed. We performed tests for trends by including the observation year as a continuous variable in a linear or logistic regression model. The sex-specific multivariate-adjusted prevalence of hyperuricaemia from 2010 through 2019 were estimated using logistic regression models after adjustment for age, BMI, eGFR, hypertension, diabetes and dyslipidaemia. Age, BMI and eGFR were included in the logistic model as continuous variables, whereas hypertension, diabetes and dyslipidaemia were included as dichotomous variables. The Stata commands used to estimate the adjusted hyperuricaemia prevalence were 'logit' and 'margins' in this study. All statistical analyses were performed using Stata V.12.0 (StataCorp LP, College Station, Texas, USA). Graphs were drawn using R software (V.3.6.1) with an available package: ggplot2. Two-sided $\mathrm{p}<0.05$ was considered statistically significant.

\section{RESULTS}

\section{The crude prevalence of hyperuricaemia in $\mathbf{2 0 1 9}$}

Table 1 showed crude prevalence of hyperuricaemia and SUA levels in participants aged $\geq 20$ years during the year 2019. A total of 66998 men and 48385 women in 2019 were included, with an average age of $42.0 \pm 12.6$ years. The overall hyperuricaemia prevalence was $25.8 \%$. The crude prevalence of hyperuricaemia and SUA levels in men were significantly higher than those in women (36.6\% vs $10.8 \%, 388.0$ (338.0-445.2) umol/L vs 270.0 $(233.0-314.0)$ umol/L; both $\mathrm{p}<0.05))$. Hyperuricaemia prevalence was around $9.0 \%$ in women aged $<50$ years and it rapidly increased with advancing age in women aged $\geq 50$ years, with the highest prevalence of $26.1 \%$ for women aged $\geq 70$ years. The burden of hyperuricaemia among men was high across all age groups and it was particularly marked in young men (39.3\% for 20-29 years and $40.5 \%$ for $30-39$ years). Hyperuricaemia prevalence and SUA levels dramatically increased with elevating BMI in both sexes $(\mathrm{p}<0.05)$; the prevalence $(95 \% \mathrm{CI})$ was $55.9 \%(55.0 \%$ to $56.9 \%)$ in obese men and $34.6 \%(32.8 \%$ to $36.5 \%)$ in obese women. In the whole population, participants with hypertension, diabetes or dyslipidaemia had significantly higher hyperuricaemia prevalence and SUA levels than the normal groups $(\mathrm{p}<0.05)$.

\section{Trends in the crude prevalence of hyperuricaemia}

A total of 671451 participants were included in the study between 2010 and 2019. As shown in table 2, the crude prevalence of hyperuricaemia significantly increased over the years in both men and women $(p<0.05)$. SUA levels among men and women gradually increased from 358.0 (313.0-407.0) umol/L and 250.0 (217.0-288.0) $\mathrm{umol} / \mathrm{L}$ in 2010 to $388.0(338.0-445.2) \mathrm{umol} / \mathrm{L}$ and 270.0
(233.0-314.0) umol/L in 2019, respectively $(\mathrm{p}<0.05)$. A significantly increasing trend in hyperuricaemia prevalence was observed during the observation period in each age category of both sexes (figure 1). The prevalence increased most sharply among participants aged 20-39 years. It increased from $22.5 \%$ (21.6\% to $23.3 \%)$ in 2010 to $40.1 \%$ (39.6\% to $40.6 \%$ ) in 2019 among young men, whereas among young women it increased from $2.5 \%$ (2.1\% to $2.9 \%$ ) in 2010 to $9.0 \%$ (8.6\% to $9.4 \%$ ) in 2019 .

\section{Trends in multivariate-adjusted prevalence of hyperuricaemia}

The multivariate-adjusted prevalence of hyperuricaemia was calculated using logistic regression models. Figure 2 showed an increasing trend in multivariate-adjusted prevalence of hyperuricaemia during the observation years in both sexes $(\mathrm{p}<0.05)$. The prevalence among men was $26.1 \%$ (25.4\% to $26.7 \%$ ) in $2010,30.9 \%$ (30.4\% to $31.4 \%$ ) in 2015 and $34.4 \%$ (34.1\% to $34.8 \%$ ) in 2019 , while among women it was $5.8 \%$ (5.4\% to $6.2 \%)$ in 2010 , $7.2 \%(6.9 \%$ to $7.5 \%)$ in 2015 and $10.1 \%$ (9.9\% to $10.3 \%$ ) in 2019 .

\section{DISCUSSION}

The present study revealed that hyperuricaemia was highly prevalent (36.6\% in men and $10.8 \%$ in women) among adults in Wuhan city, China, during 2019. The burden of hyperuricaemia among men was substantial in all age groups. Hyperuricaemia prevalence varied distinctly by sex, age, BMI, hypertension, diabetes and dyslipidaemia, with young men, old women and participants with metabolic disorders having a significantly higher prevalence.

This study also investigated the trend of hyperuricaemia over a decade period (from 2010 to 2019) and revealed a significantly increasing trend in multivariateadjusted prevalence of hyperuricaemia in both sexes. Moreover, it was observed that the prevalence increased most sharply among young adults during the observation period, which meant that hyperuricaemia occurred more and more frequently in young adults.

The estimated prevalence in our study (25.8\% in 2019) was much higher than those reported in America $(20.1 \%$ in $2015-2016),{ }^{16}$ Italy $(11.9 \%$ in 2009$),{ }^{13}$ Korea $(11.4 \%$ in $2016)^{31}$ and a previous national survey in China $(13.0 \%$ in 2007-2011). ${ }^{18}$ A cross-sectional study from Bangkok, Thailand, used data of the annual physical examination and reported a prevalence rate of $24.4 \%$ in urban residents, ${ }^{32}$ which was close to our result. Epidemiological studies demonstrated that urban individuals had a higher prevalence of hyperuricaemia than rural residents. ${ }^{17} 19$ In the present study, almost all the participants included were urban citizens, which may help explain the high hyperuricaemia prevalence.

Overweight or obesity was a well-accepted risk factor for hyperuricaemia, ${ }^{33}$ which was validated in our study. We found that hyperuricaemia prevalence increased significantly with elevating BMI in both sexes. Based on information in table 1 , it could be calculated that nearly 


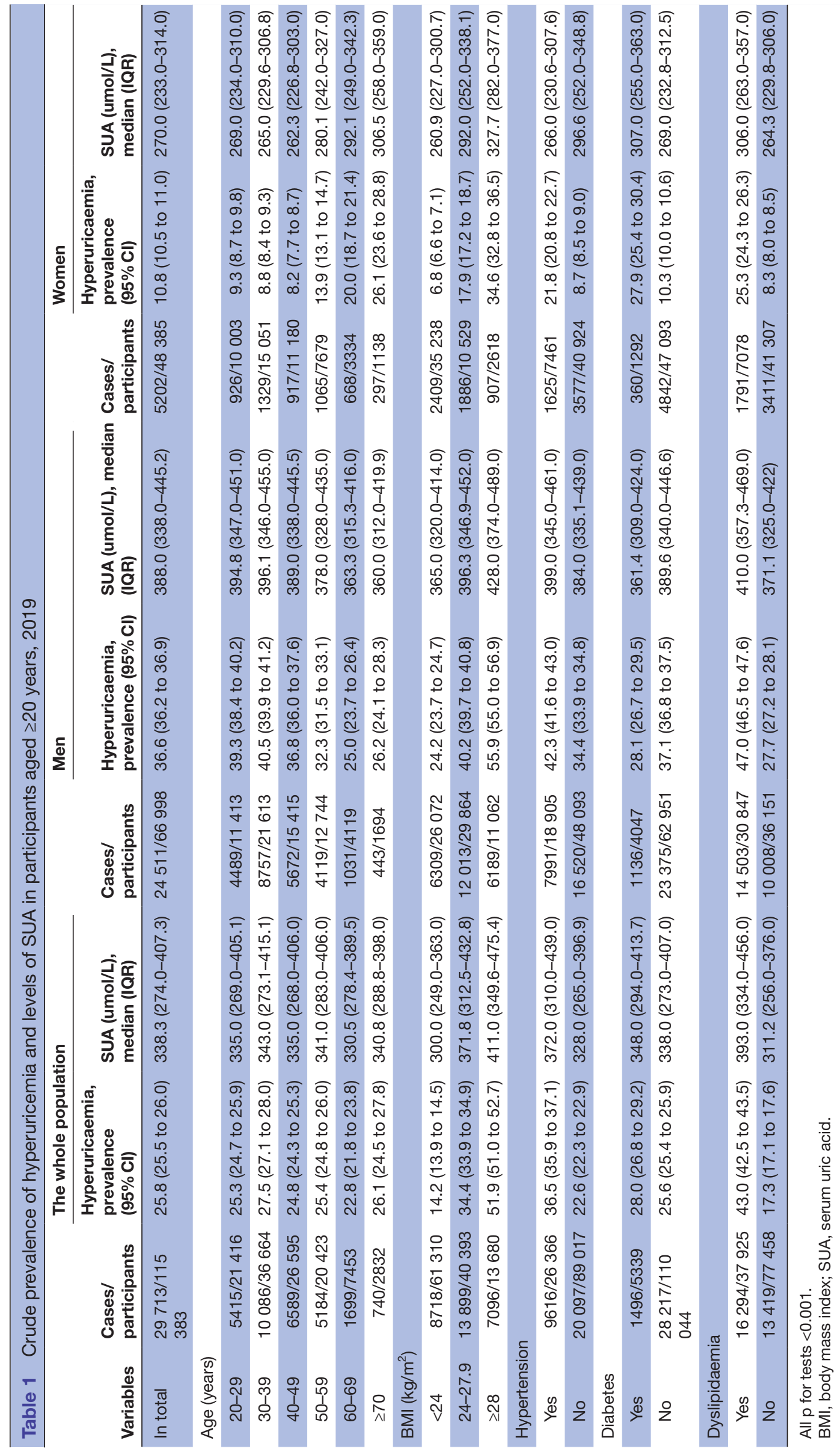




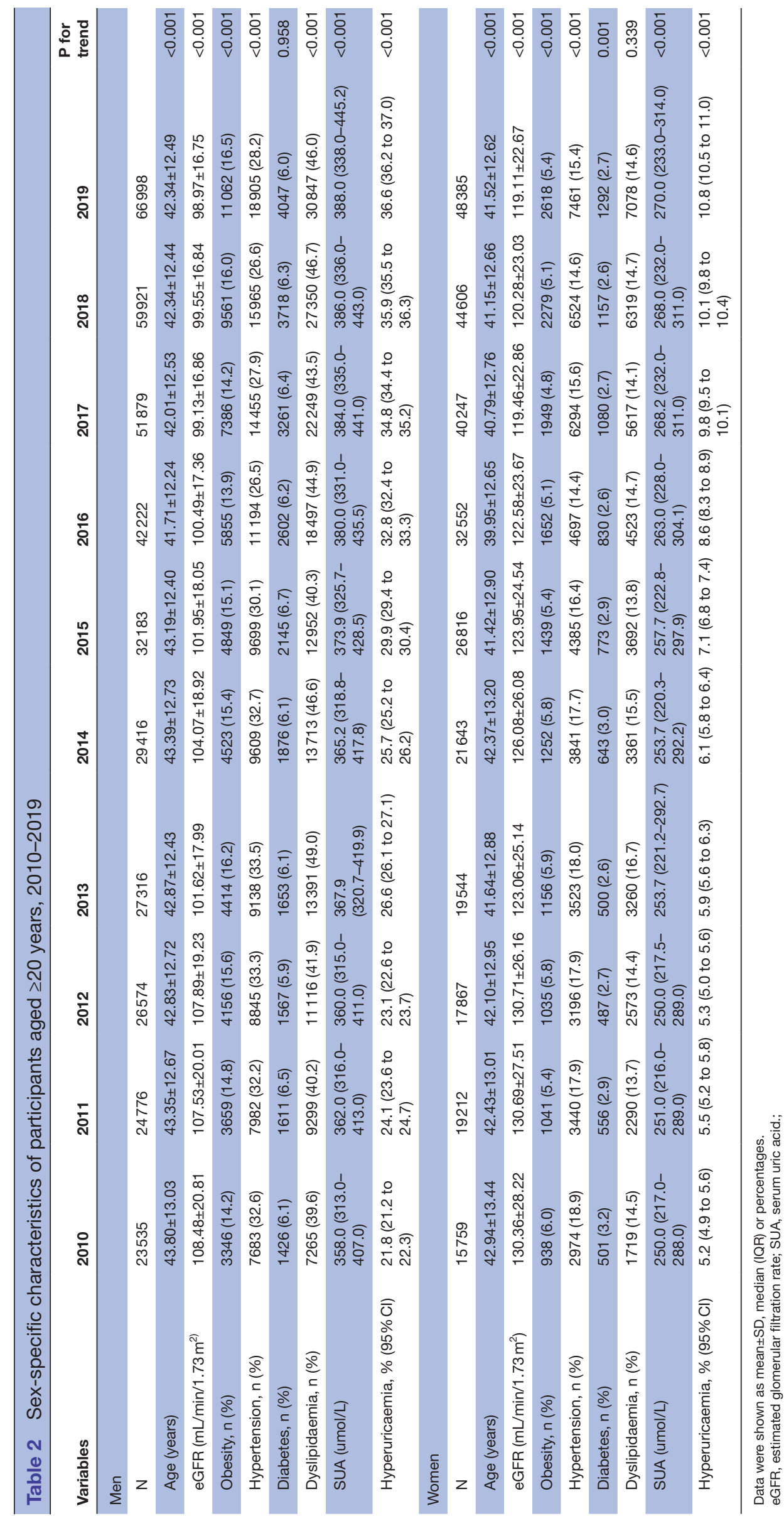




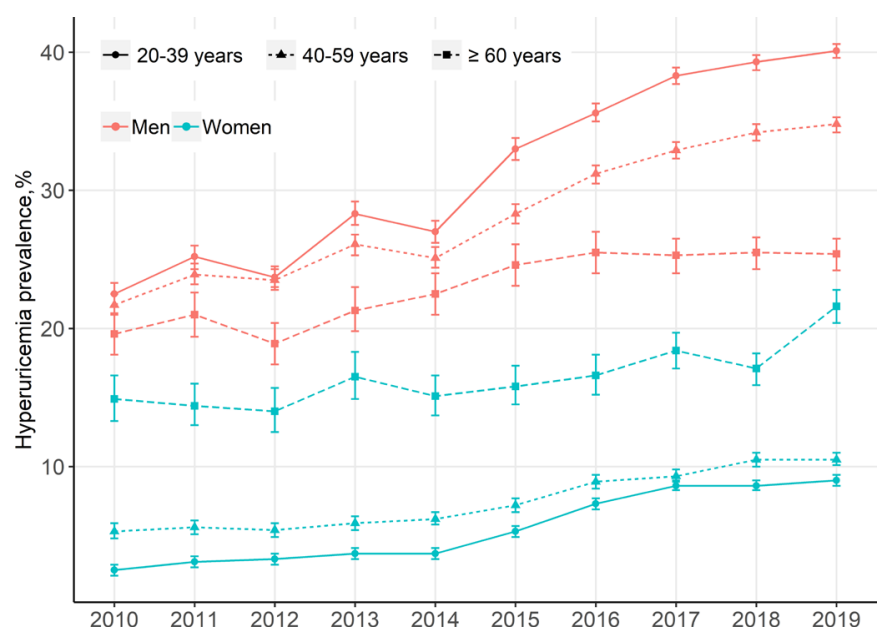

Figure 1 Sex-specific and age-specific trends in hyperuricaemia prevalence (95\% Cl), 2010-2019.

$61.0 \%$ of men were overweight or obese and $44.5 \%$ of them were identified as hyperuricaemia. The prevalence of overweight or obesity among men was reported to be $38.6 \%$ in a representative sample of Wuhan community residents aged 15-69 years. ${ }^{34}$ Compared with this representative sample, the working population in the present study had markedly higher ratios of men, labour force and overweight or obesity. The heavy burden of overweight or obesity may be another reason to explain the highly prevalent hyperuricaemia. It was also observed that hyperuricaemia was closely correlated with hypertension, diabetes and dyslipidaemia, which accorded with the previous studies. ${ }^{6-9}$ These metabolic disorders were common comorbidities in participants with hyperuricaemia, leading to higher risk of renal and cardiovascular diseases.

The present study found that hyperuricaemia prevalence was higher among women aged $\geq 50$ years and it further increased with advancing age. Several studies from Asian and European countries revealed a roughly positive

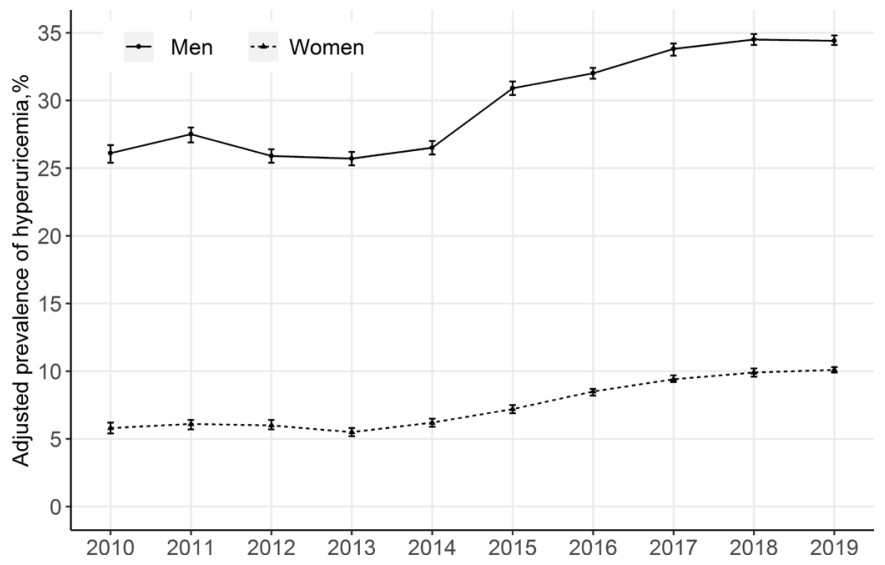

Figure 2 Sex-specific trends in multivariate-adjusted hyperuricaemia prevalence $(95 \% \mathrm{Cl})$ among participants aged $\geq 20$ years, 2010-2019. The prevalence was adjusted for age, body mass index, estimated glomerular filtration rate, hypertension, diabetes and dyslipidaemia. association of hyperuricaemia prevalence with age among women, ${ }^{13} 141831$ which accorded with our results. Given the huge sex difference in hyperuricaemia prevalence, we thought that sex hormones may play a key role. One explanation was that female sex hormones had protective effects against hyperuricaemia. A cross-sectional study of 58870 South Korean women demonstrated that hyperuricaemia prevalence significantly increased with the menopausal stage, after controlling for potential confounders. ${ }^{35}$ Postmenopausal women are characterised by materially declined levels of female sex hormones (especially estradiol and progesterone). When they grow older, levels of estradiol and progesterone would decline further. The BioCycle study demonstrated that SUA levels were inversely associated with these two hormones. ${ }^{36}$ Until now, how estradiol and progesterone lower SUA levels was not fully understood. They probably effect via promoting renal excretion of uric acid. ${ }^{37} 38$ Another two explanations would be the higher prevalence of obesity and alcohol drinking in men than women, ${ }^{34} 39$ as obesity and alcohol drinking are well-established risk factors for hyperuricaemia.

Data from the National Health and Nutrition Examination Survey demonstrated that hyperuricaemia prevalence among American adults significantly increased from $18.2 \%$ in $1988-1994$ to $21.4 \%$ in $2007-2008$ and concluded that the increasing trend of hyperuricaemia was likely due to rising prevalence of obesity and hypertension. ${ }^{12}$ However, in the present study, we did not observe an increasing trend in obesity and hypertension over the years (table 2). Thus, it might be some other factors responsible for the increasing prevalence of hyperuricaemia among our study participants. We thought that gradually westernised dietary structure and rising consumption of fructose-sweetened soft drinks might be the main causes. Western diets contained much more purine than the traditional Chinese diets, leading to a higher risk of developing hyperuricaemia. In addition, accumulating evidence showed that fructose-sweetened drinks, although containing no purines, could induce hyperuricaemia. ${ }^{40-42}$ Fructose intake per capita has dramatically increased during the past few decades, ${ }^{43-45}$ in parallel with the increasing burden of hyperuricaemia. Alcohol consumption and several lifestyle habits like inactivity and sedentary behaviours have been established as risk factors for hyperuricaemia. There are no studies directly investigating the associations between these risk factors and the increasing trends in hyperuricaemia prevalence. However, findings from the China Kadoorie Biobank reported a modest increase in alcohol consumption, drinking frequency and heavy episodic drinking prevalence among men in the past decade, particularly among the young men, ${ }^{39}$ which may help explain our results.

To the best of our knowledge, the present study first revealed age-specific trends in hyperuricaemia over a decade among Chinese adults and found that hyperuricaemia prevalence increased most sharply among young 
adults during the observation period. A large analysis of 128014 Irish adults revealed an increasing trend in hyperuricaemia from 2006 through 2014 across all age groups, with the most increment among young participants aged 18-39 years ${ }^{14}$; a finding that was similar to our result. In addition to hyperuricaemia, several hyperuricaemiarelated diseases such as diabetes and cardiovascular events also occurred more frequently among young adults over the past years, ${ }^{4647}$ posing a serious threat to public health. Based on the trend revealed in our study, hyperuricaemia prevalence was much likely to continue rising in the coming years. Policymakers should pay more attention to the burden of hyperuricaemia, especially among young adults.

The strengths of the present study were distinct. This study included a large sample size of participants (more than 730000 adults) from the general population, which made our findings more convincible. In addition, to the best of our knowledge, this was the first study to investigate the trend in hyperuricaemia prevalence over the recent decade (2010-2019) among adults in Wuhan city, contributing to the management of hyperuricaemia in this area.

The study also had several limitations. First, as the participants in the present study were recruited from a health management centre, selection biases could not be avoided. The participants may be not a representative sample of the general population in community. Therefore, it should take caution to interpret the findings of this study. In fact, as almost all the participants were urban citizens working in government organisations or enterprises across every district of Wuhan city, the study participants were more likely a sample of working populations than community residents. Additionally, we estimated the hyperuricaemia prevalence after controlling several confounders (shown in figure 2), which would help reduce the bias through statistical analyses. Second, hyperuricaemia prevalence may be underestimated in our study. We diagnose hyperuricaemia only according to SUA levels. However, participants with hyperuricaemia might have normal SUA levels if they were undergoing SUA-lowering therapies. Third, our study did not collect data on diets and lifestyles that were related to hyperuricaemia. Changes of these variables such as fructose intake over the years may help explain the trends in hyperuricaemia. Fourth, in the study population, there existed participants who underwent health check-ups more than once during the study period. Their multiple medical records were included in the analyses, which may finally influence the accuracy of our results. Fifth, the definition of hyperuricaemia highlights the measurement of SUA on two different days under the normal purine diet. However, SUA was measured only once in the present study, which would affect the screening rate of hyperuricaemia.

\section{Conclusions}

In summary, a high burden of hyperuricaemia was found among adults in Wuhan city. Moreover, hyperuricaemia occurred more and more frequently in young adults. Our study also revealed a significant increasing trend in multivariate-adjusted prevalence of hyperuricaemia among adults from 2010 through 2019. Promoting dietary change, weight loss and physical activity in community or workplace would be effective measures to prevent and control hyperuricaemia. These measures should be taken urgently, especially among young adults, postmenopausal women, and participants with metabolic disorders, for they were at higher risks.

Acknowledgements The authors want to thank the participants in the Health Management Center of Tongji Hospital for participating in this present study.

Contributors All authors are in agreement with the content of the manuscript. ZW designed the study and drafted the article. LS, LH and YL contributed to the conception, analysis and critically revised the manuscript. $\mathrm{XL}$ and $\mathrm{YH}$ participated in the data collection and revised the manuscript.

Funding The authors have not declared a specific grant for this research from any funding agency in the public, commercial or not-for-profit sectors.

Competing interests None declared.

Patient consent for publication Not required.

Ethics approval The study was conducted in accordance with the Declaration of Helsinki and it was approved by the ethics committee of Tongji Hospital, Tongji Medical College, Huazhong University of Science and Technology with a waiver of informed consent. The Institutional Review Board approval number was TJ-IRB20200513.

Provenance and peer review Not commissioned; externally peer reviewed.

Data availability statement Data are available upon reasonable request.

Supplemental material This content has been supplied by the author(s). It has not been vetted by BMJ Publishing Group Limited (BMJ) and may not have been peer-reviewed. Any opinions or recommendations discussed are solely those of the author(s) and are not endorsed by BMJ. BMJ disclaims all liability and responsibility arising from any reliance placed on the content. Where the content includes any translated material, BMJ does not warrant the accuracy and reliability of the translations (including but not limited to local regulations, clinical guidelines, terminology, drug names and drug dosages), and is not responsible for any error and/or omissions arising from translation and adaptation or otherwise.

Open access This is an open access article distributed in accordance with the Creative Commons Attribution Non Commercial (CC BY-NC 4.0) license, which permits others to distribute, remix, adapt, build upon this work non-commercially, and license their derivative works on different terms, provided the original work is properly cited, appropriate credit is given, any changes made indicated, and the use is non-commercial. See: http://creativecommons.org/licenses/by-nc/4.0/.

ORCID iD

Yongman Lv http://orcid.org/0000-0002-3525-492X

\section{REFERENCES}

1 Dalbeth N, Merriman TR, Stamp LK. Gout. Lancet 2016;388:2039-52.

2 Lee C-L, Tsai S-F. Association between mortality and serum uric acid levels in non-diabetes-related chronic kidney disease: an analysis of the National health and nutrition examination survey, USA, 19992010. Sci Rep 2020;10:17585.

3 Waheed YA, Yang F, Sun D. The role of asymptomatic hyperuricemia in the progression of chronic kidney disease CKD and cardiovascular diseases CVD. Korean J Intern Med 2020 doi:10.3904/kjim.2020.340

4 Hong JW, Noh JH, Kim D-J. Association between serum uric acid and spirometric pulmonary function in Korean adults: the 2016 Korea National health and nutrition examination survey. PLoS One 2020;15:e0240987.

5 Ali N, Perveen R, Rahman S, et al. Prevalence of hyperuricemia and the relationship between serum uric acid and obesity: a study on Bangladeshi adults. PLoS One 2018;13:e0206850.

6 Haque T, Rahman S, Islam S, et al. Assessment of the relationship between serum uric acid and glucose levels in healthy, prediabetic and diabetic individuals. Diabetol Metab Syndr 2019;11:49. 
7 Ali N, Rahman S, Islam S, et al. The relationship between serum uric acid and lipid profile in Bangladeshi adults. BMC Cardiovasc Disord 2019;19:42

8 Ali N, Mahmood S, Islam F, et al. Relationship between serum uric acid and hypertension: a cross-sectional study in Bangladeshi adults. Sci Rep 2019;9:9061.

9 Ali N, Miah R, Hasan M, et al. Association between serum uric acid and metabolic syndrome: a cross-sectional study in Bangladeshi adults. Sci Rep 2020;10:7841.

10 GBD 2017 Causes of Death Collaborators. Global, regional, and national age-sex-specific mortality for 282 causes of death in 195 countries and territories, 1980-2017: a systematic analysis for the global burden of disease study 2017. Lancet 2018;392:1736-88.

11 GBD 2017 DALYs and HALE Collaborators. Global, regional, and national disability-adjusted life-years (DALYs) for 359 diseases and injuries and healthy life expectancy (HALE) for 195 countries and territories, 1990-2017: a systematic analysis for the global burden of disease study 2017. Lancet 2018;392:1859-922.

12 Zhu Y, Pandya BJ, Choi HK. Prevalence of gout and hyperuricemia in the US general population: the National health and nutrition examination survey 2007-2008. Arthritis Rheum 2011;63:3136-41.

13 Trifirò G, Morabito P, Cavagna L, et al. Epidemiology of gout and hyperuricaemia in Italy during the years 2005-2009: a nationwide population-based study. Ann Rheum Dis 2013;72:694-700.

14 Kumar A, Browne LD, Li X, et al. Temporal trends in hyperuricaemia in the Irish health system from 2006-2014: a cohort study. PLoS One 2018;13:e0198197.

15 Kuo C-F, Grainge MJ, Zhang W, et al. Global epidemiology of gout: prevalence, incidence and risk factors. Nat Rev Rheumatol 2015;11:649-62.

16 Chen-Xu M, Yokose C, Rai SK, et al. Contemporary prevalence of gout and hyperuricemia in the United States and Decadal trends: the National health and nutrition examination survey, 2007-2016. Arthritis Rheumatol 2019;71:991-9.

17 Liu H, Zhang X-M, Wang Y-L, et al. Prevalence of hyperuricemia among Chinese adults: a national cross-sectional survey using multistage, stratified sampling. J Nephrol 2014;27:653-8.

18 Wu J, Qiu L, Cheng X-Q, et al. Hyperuricemia and clustering of cardiovascular risk factors in the Chinese adult population. Sci Rep 2017;7:5456

19 Song $\mathrm{P}$, Wang $\mathrm{H}$, Xia W, et al. Prevalence and correlates of hyperuricemia in the middle-aged and older adults in China. Sci Rep 2018;8:4314.

20 Liu R, Han C, Wu D, et al. Prevalence of hyperuricemia and gout in mainland China from 2000 to 2014: a systematic review and metaanalysis. Biomed Res Int 2015;2015:762820

21 Statistics Bureau of Wuhan Municipality. Wuhan statistical yearbook 2010. Beijing: China Statistics Press, 2010.

22 Statistics Bureau of Wuhan Municipality. Wuhan statistical yearbook 2018. Beijing: China Statistics Press, 2018.

23 Lao XQ, Ma WJ, Sobko T, et al. Dramatic escalation in metabolic syndrome and cardiovascular risk in a Chinese population experiencing rapid economic development. BMC Public Health 2014;14:983.

24 Misra A, Khurana L. Obesity and the metabolic syndrome in developing countries. J Clin Endocrinol Metab 2008;93:s9-30.

25 Flegal KM, Kruszon-Moran D, Carroll MD, et al. Trends in obesity among adults in the United States, 2005 to 2014. JAMA 2016;315:2284-91.

26 von Elm E, Altman DG, Egger M, et al. The strengthening the reporting of observational studies in epidemiology (STROBE) statement: guidelines for reporting observational studies.

27 Hochberg MC, Smolen JS, Weinblatt ME. Rheumatology. 3rd edn. New York: Mosby, 2003.

28 Feig DI, Kang D-H, Johnson RJ. Uric acid and cardiovascular risk. $N$ Engl J Med 2008;359:1811-21.
29 Bureau of Disease Control, Ministry of Health of the People's Republic of China. Guidelines for prevention and control of overweight and obesity in Chinese adults. Beijing: People's Medical Publishing House, 2006.

30 Joint Committee Issued Chinese Guideline for the Management of Dyslipidemia in Adults. Chinese guideline for the management of dyslipidemia in adults. Chinese $J$ Cardiol 2016;44:833-53.

31 Kim Y, Kang J, Kim G-T. Prevalence of hyperuricemia and its associated factors in the general Korean population: an analysis of a population-based nationally representative sample. Clin Rheumatol 2018;37:2529-38.

32 Uaratanawong S, Suraamornkul S, Angkeaw S, et al. Prevalence of hyperuricemia in Bangkok population. Clin Rheumatol 2011;30:887-93.

33 Zhang N, Chang Y, Guo X, et al. A body shape index and body roundness index: two new body indices for detecting association between obesity and hyperuricemia in rural area of China. Eur $J$ Intern Med 2016;29:32-6.

34 Zhong Q, YL L, Huang YX, et al. Prevalence and influencing factors of overweight and obesity among community residents of Wuhan City, 2018. Chin J Public Health 2019;35:1210-4.

35 Cho SK, Winkler CA, Lee S-J, et al. The prevalence of hyperuricemia sharply increases from the late menopausal transition stage in middle-aged women. J Clin Med 2019;8:296. doi:10.3390/ jcm8030296

36 Mumford SL, Dasharathy SS, Pollack AZ, et al. Serum uric acid in relation to endogenous reproductive hormones during the menstrual cycle: findings from the BioCycle study. Hum Reprod 2013;28:1853-62.

37 Antón FM, García Puig J, Ramos T, et al. Sex differences in uric acid metabolism in adults: evidence for a lack of influence of estradiol-17 beta (E2) on the renal handling of urate. Metabolism 1986;35:343-8.

38 Yahyaoui R, Esteva I, Haro-Mora JJ, et al. Effect of long-term administration of cross-sex hormone therapy on serum and urinary uric acid in transsexual persons. J Clin Endocrinol Metab 2008;93:2230-3.

39 Im PK, Millwood IY, Guo Y, et al. Patterns and trends of alcohol consumption in rural and urban areas of China: findings from the China Kadoorie Biobank. BMC Public Health 2019;19:217.

40 Lecoultre V, Egli L, Theytaz F, et al. Fructose-induced hyperuricemia is associated with a decreased renal uric acid excretion in humans. Diabetes Care 2013;36:e149-50.

41 Caliceti C, Calabria D, Roda A, et al. Fructose intake, serum uric acid, and cardiometabolic disorders: a critical review. Nutrients 2017;9 doi:10.3390/nu9040395

42 Ebrahimpour-Koujan S, Saneei P, Larijani B, et al. Consumption of sugar sweetened beverages and dietary fructose in relation to risk of gout and hyperuricemia: a systematic review and meta-analysis. Crit Rev Food Sci Nutr 2020:60:1-10.

43 Rho YH, Zhu Y, Choi HK. The epidemiology of uric acid and fructose. Semin Nephrol 2011;31:410-9.

44 Bray GA, Nielsen SJ, Popkin BM. Consumption of high-fructose corn syrup in beverages may play a role in the epidemic of obesity. Am J Clin Nutr 2004:79:537-43.

45 Johnson RJ, Segal MS, Sautin Y, et al. Potential role of sugar (fructose) in the epidemic of hypertension, obesity and the metabolic syndrome, diabetes, kidney disease, and cardiovascular disease. Am J Clin Nutr 2007;86:899-906.

46 Menke A, Casagrande S, Geiss L, et al. Prevalence of and trends in diabetes among adults in the United States, 1988-2012. JAMA 2015;314:1021-9.

47 Sarink D, Nedkoff L, Briffa T, et al. Trends in age- and sex-specific prevalence and incidence of cardiovascular disease in Western Australia. Eur J Prev Cardiol 2018;25:1280-90. 